\title{
A Small Patch Antenna with Ellipse Disc Loaded/cut by Using Differential Evolution
}

\author{
Qing Zhang ${ }^{1,}$, , Sanyou Zeng ${ }^{2, b}$ \\ ${ }^{1}$ Huanggang Normal University, Huangzhou, Hubei, China \\ ${ }^{2}$ China University of Geosciences, Wuhan, Hubei, China \\ a823963996@qq.com, bsanyouzeng@gmail.com
}

\begin{abstract}
Keywords: Multisim, computer hardware, virtual simulation
Abstract. Computer hardware course is abstract, Multisim simulation software will be integrated into the computer hardware course teaching, promote the teaching to the development of information technology through the simulation play the role of technology, brings to the teachers and students professional and comprehensive virtual experiment environment. This paper first introduces the basic situation of Multisim software platform, combined with the practice of the current computer hardware curriculum system of computer composition principle of this course, analyzes the composition of the functional units of the computer virtual simulation design and implementation method, discusses the important role of the virtual simulation technology platform, a comprehensive system of value of the virtual simulation the hardware based on Multisim.
\end{abstract}

\section{Introduction}

Evolutionary antenna design has been investigated by researchers since the early 1990s, e.g., [1], [2], [3]. And it is still a hot topic up to date. For example, [4], [5], etc. designed wire antennas; [6], [7], [8], etc. designed planar antennas; [10], [11], [9], [12], [13], etc. designed antenna arrays. Once a new evolutionary algorithm is proposed, it is usually applied to design antenna soon. Particle swarm optimization (PSO) [14] is used to design antennas, e.g., [15], [16], [17]. Differential evolution (DE) [18] is adopted to design antennas, e.g., [6], [19], [20]. Evolutionary algorithm with machine learning technique is a hot research topic in the evolutionary computation area, e.g., [21]. The achievements are employed to design antennas as well, e.g., [6]. In this paper, a patch antenna in the $2.45 \mathrm{GHz}$ band is designed by using differential evolution. After a brief review of the evolutionary antennas in Section I, this paper is organized as follows: Section II introduces the concepts of constrained optimization problems (COPs) and a typical evolutionary algorithm, differential evolution. Section III models the antenna design as a COP. Then the antenna design problem is solved by DE in Section IV. Finally, Section V summarizes the conclusion of this paper.

\section{Preliminary}

A. Constrained Optimization Problem

Antenna design can be modeled as a constrained optimization problem (COP). We review the COP in this subsection. Without loss of generality, minimization optimization is assumed.

Definition 1: (Constrained Optimization Problem(COP)) A general COP includes a set of $\mathrm{n}$ variables, an objective function, and a set of $\mathrm{m}$ constraints. Objective function and constraints are functions of the variables. The optimization goal is to

$$
\begin{aligned}
& \min \quad \frac{y=f(\vec{x})}{g(\vec{x})}=\left(g_{1}(\vec{x}), g_{2}(\vec{x}), \ldots, g_{m}(\vec{x})\right) \leq \overrightarrow{0} \\
& \text { st: } \\
& \text { where } \\
& \quad \quad \mathbf{X}=\{\vec{x} \mid \leq \vec{x} \leq \vec{u}\} \\
& \quad \vec{l}=\left(l_{1}, l_{2}, \ldots, l_{n}\right), \vec{u}=\left(u_{1}, u_{2}, \ldots, u_{n}\right)
\end{aligned}
$$


where $\vec{x}$ is the solution vector (solution for short) and $\mathbf{X}$ denotes the solution space, $\vec{l}$ and $\vec{u}$ are the lower bound and upper bound of the solution space, $g(\vec{x}) \leq 0$ is the constraint and $\overrightarrow{0}$ denotes the constrained boundary.

The antenna design in the paper is solved by our evolutionary antenna solver. The evolutionary algorithms in the solver demands a normalized solution space from $X=[\vec{\imath}, \vec{u}]$ to $X$ by

$$
{\overrightarrow{x^{\prime}}}_{j}=\frac{\vec{x}_{j}-\vec{l}_{j}}{\vec{u}_{j}-\vec{l}_{j}}, j=1,2, \ldots, n
$$

The violation of a constraint is usually evaluated as

$$
G_{i}(\vec{x})=\max \left\{g_{i}(\vec{x}), 0\right\}, i=1,2, \ldots, m \text {. }
$$

The constraint-violation of a solution is defined as the average of the normalized violations of all constraints of the solution in the following:

Definition 2: (Constraint-violation)

$$
(\vec{x})=\frac{1}{m} \sum_{i=1}^{m} \frac{G_{i}(\vec{x})}{\max _{\vec{x} \in \mathbf{P}(0)}\left\{G_{i}(\vec{x})\right\}}
$$

B. Differential Evolution

The differential evolution (DE) [18] is typically effective in solving continuous problems including continuous COPs. The DE is adopted to solve antenna design problems in this paper. It has many different schemes to generate offsprings. The basic DE scheme DE/rand/1/bin is used in the paper. A version of the algorithm is described in Algorithm II-B.

In the affine mutation operator, the offspring $U i(j)$ may exceed the normalized range $[0,1]$. We set $\operatorname{Ui}(\mathrm{j})=$ fraction $(\operatorname{Ui}(\mathrm{j}))$ when $\operatorname{Ui}(\mathrm{j})<0.0$, and $\operatorname{Ui}(\mathrm{j})=1-$ fraction $(\operatorname{Ui}(\mathrm{j}))$ when $\operatorname{Ui}(\mathrm{j})>1.0$. fraction $(\operatorname{Ui}(\mathrm{j}))$ returns the fraction part of $\operatorname{Ui}(\mathrm{j})$, e.g., fraction $(-1.25)=0.25$, fraction $(1.25)=0.25$. Ui better than $\mathrm{Pi}$ means that $\mathrm{cv}(\mathrm{Ui})<\mathrm{cv}(\mathrm{Pi})$ or that $\mathrm{cv}(\mathrm{Ui})==\mathrm{cv}(\mathrm{Pi})$ and $\mathrm{f}(\mathrm{Ui})<\mathrm{f}(\mathrm{Pi})$.

\section{MODELING THE ANTENNA DESIGN AS A COP}

In this section, the specifications of the antenna is first given. And following Eq. (1), a COP problem is constructed based on the specifications. Then the DE algorithm can be used to solve the constructed COP. Consequently, the antenna design problem will be solved.

A. Design specifications of the Antenna

An omnidirectional microstrip antenna is intended to be designed in a range of $2.45 \mathrm{GHz}$ band. The specifications are shown in Table I.

TABLE I

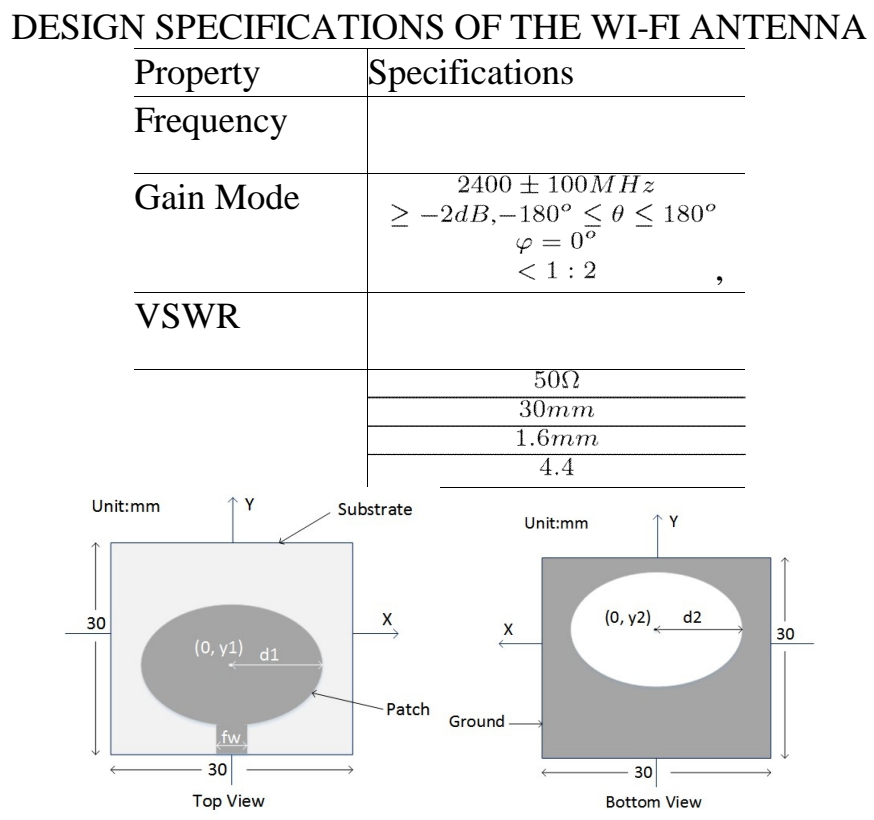

Fig. 1. Geometric shape of the antenna 


\section{B. Antenna design formed as a COP}

The geometric structure of the antenna is shown in Fig. 1. A square flame retardant 4(FR4) substrate is given with side $30 \mathrm{~mm}$ and with thickness $1.6 \mathrm{~mm}$ and the relative permittivity is 4.4 . An ellipse disc patch is loaded on the top of the substrate while the ground is cut off an ellipse disc on the bottom.

The centers of the two ellipse discs are located right above/on $y$-axis ( denoted as $(0, y 1,0)$ and $(0, \mathrm{y} 2,1.6)$ respectively), each has an axis parallel to $\mathrm{x}$-axis ( denoted as $\mathrm{d} 1$ and $\mathrm{d} 2$ respectively), and the micro-strip feed line connected the top patch goes along y-axis (the width of the feed line is denoted as fw). Suppose both ellipse have same axis ratio (denoted as r).

The above six parameters $\mathrm{y} 1, \mathrm{y} 2, \mathrm{~d} 1, \mathrm{~d} 2, \mathrm{fw}, \mathrm{r}$ are design variables in this antenna design. That is, the solution vector is $\vec{x}=(\mathrm{y} 1, \mathrm{y} 2, \mathrm{~d} 1, \mathrm{~d} 2, \mathrm{fw}, \mathrm{r})$. Based on the space constraints in the specifications, the solution space is supposed in the following ranges.

$-14.0 \mathrm{~mm} \leq \mathrm{y} 1 \leq 14.0 \mathrm{~mm},-14.0 \mathrm{~mm} \leq \mathrm{y} 2 \leq 14.0 \mathrm{~mm}, 1.0 \mathrm{~mm} \leq \mathrm{d} 1 \leq 14.0 \mathrm{~mm}, 1.0 \mathrm{~mm} \leq \mathrm{d} 2 \leq$ $14.0 \mathrm{~mm}, 1.0 \mathrm{~mm} \leq \mathrm{fw} \leq 3.0 \mathrm{~mm}$ and $0.01 \leq \mathrm{r} \leq 1.0$, i.e., the Eq. (5)

$$
\begin{aligned}
& \mathbf{X}=\{\vec{x} \mid \vec{l} \leq \vec{x} \leq \vec{u}\} \\
& \vec{x}=\left(y_{1}, y_{2}, d_{1}, d_{2}, f w, r\right) \\
& \vec{l}=(-14.0,-14.0,1.0,1.0,1.0,0.01) \\
& \vec{u}=(14.0,14.0,14.0,14.0,3.0,1.0)
\end{aligned}
$$

The objective and constraints are modeled according to the design specification in Table I. The gains are sampled in 5o increments over the region $-180^{\circ} \leq \theta \leq 180^{\circ}$ and $\varphi=0^{\circ}$, and the frequencies are sampled in $50 \mathrm{MHz}$ increments over the band from $2.35 \mathrm{GHz}$ to $2.55 \mathrm{GHz}$.

The objective is defined as the summary of all the gain variances and the VSWR variance over the frequency band, see Eq. (6).

$f(\vec{x})=\sum \sum G V$ ariance $_{\phi, \theta}+V S W R V$ ariance

The constraints on gains are defined over the interested directional region and the frequency band based on the specifications in Table I, see Eq.(7).

$$
g \operatorname{Gain}(\varphi, \theta, \operatorname{frq})(\vec{x})=-2.0-\operatorname{Gain}(\varphi, \theta, \operatorname{frq})
$$

The constraints on VSWR are defined over the frequency band, see Eq. (8).

$g V S W R_{f r q}(\vec{x})=V S W R_{f r q}-2.0$

Another kind of constraints is that the ellipse disc must stay inside of the square with side $30 \mathrm{~mm}$, which demands that

$$
\begin{aligned}
& \text { gEllipse }_{1}(\vec{x})=y_{1}+r * d_{1}-15 \\
& \text { gEllipse }_{2}(\vec{x})=y_{2}+r * d_{2}-15 \\
& \text { gEllipse }_{3}(\vec{x})=-15-\left(y_{1}-r * d_{1}\right) \\
& \text { gEllipse }_{4}(\vec{x})=-15-\left(y_{2}-r * d_{2}\right)
\end{aligned}
$$

The number of constraints on gains is $72 * 5=360$ respectively, on VSWR, the number is 5 , and on the ellipseconstraints, the number is 4 . In all, there are 369 constraints.

The antenna design is formed as a COP as follows:

$$
\begin{aligned}
& g V S W R_{f r q}(\vec{x})=V S W R_{f r q}-2.0 \leq 0 \\
& \text { gEllipse }_{1}(\vec{x})=y_{1}+r * d_{1}-15 \leq 0 \\
& \text { gEllipse }_{2}(\vec{x})=y_{2}+r * d_{2}-15 \leq 0 \\
& \text { gEllipse }_{3}(\vec{x})=-15-\left(y_{1}-r * d_{1}\right) \leq 0 \\
& \text { gEllipse }_{4}(\vec{x})=-15-\left(y_{2}-r * d_{2}\right) \leq 0
\end{aligned}
$$

Since the objective is actually the summary of all the gain variances and the VSWR variance over the frequency band, Then a antenna with stable performance over the frequency band will be preferred.

\section{The realization method of virtual technology}

The antenna design COP problem Eq. (10) is solved by using DE in this section. 


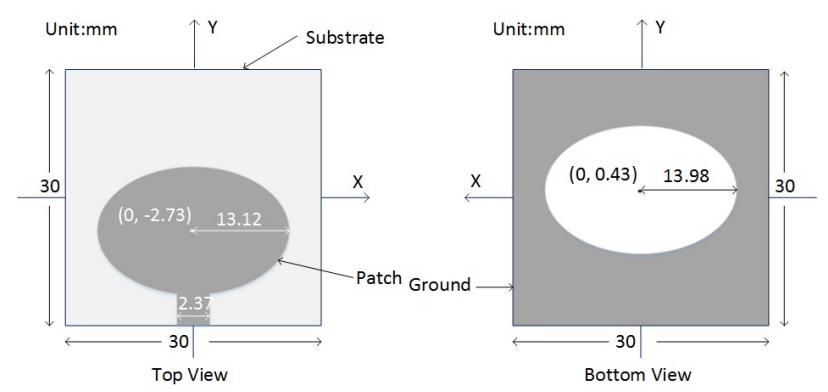

Fig. 2. Geometric structure of an evolved antenna

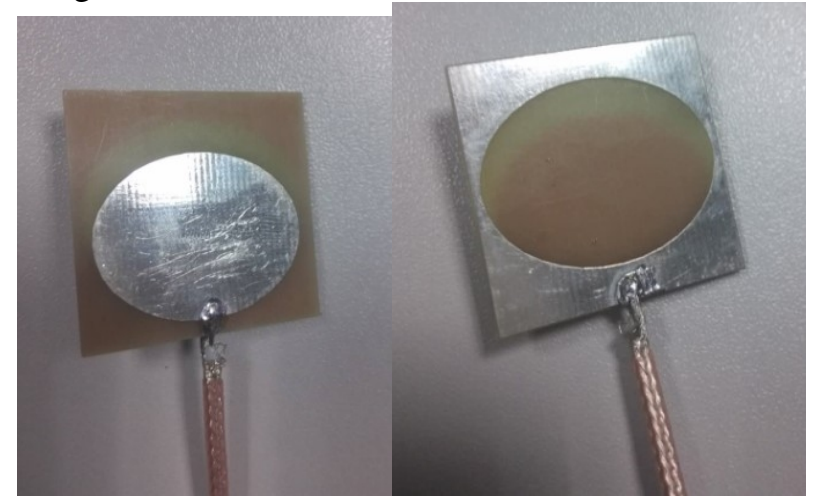

Fig. 3. Photo of the prototype of an evolved antenna

\section{A. Setting DE parameters}

The DE parameters are set as follows:

Evolutionary generations $T=500$.

Population size $N P=50$.

Crossover rate $C R=0.9$.

Scaling factor $F=\operatorname{rndreal}(0,1)$.

Uniform mutation probability $p_{m}=0.01$.

\section{B. Results and Discussion}

The electromagnetic simulation software HFSS is adopted for evaluating the performance of the antenna during the run of the DE. Some antennas have been obtained. Fig. 2 shows the geometric structure of an evolved antenna. A photo of the prototype is shown in Fig. 3.

We have measured the S-parameters( VSWRs ) of the evolved antenna. And the comparison result of S-parameters over the frequency range from $2.35 \mathrm{GHz}$ to $2.55 \mathrm{GHz}$ between the simulated and the measured are pictured in Fig. 4. The measured S-parameters are matched the simulated Sparameters, and they are all lower than -10dB (i.e., VSWR lower than 2), see Fig. 4.

However, the gains were not measured because of the expensive cost. The graph of the simulated gains over the range $-180^{\circ} \leq \theta \leq 180^{\circ}, \phi=9^{\circ}$ at $2.45 \mathrm{GHz}$ frequency is shown in Fig. 5. It can be observed that the simulated gains matched the specifications on the whole.

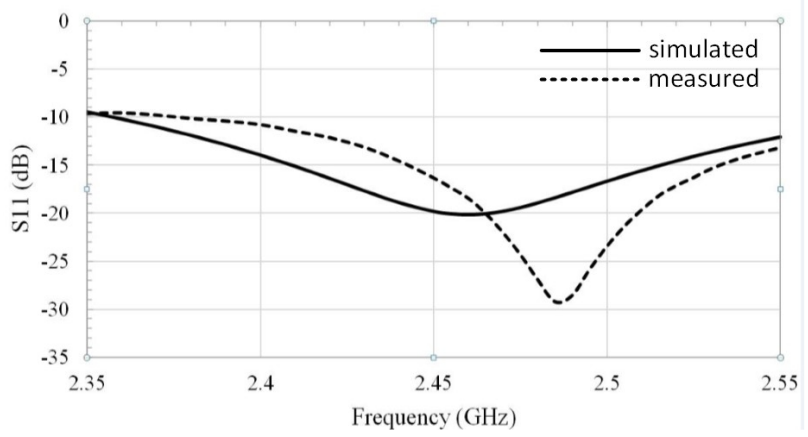

Fig. 4. Comparison of S-parameter $(V S W R)$ between the simulated and the measured of the evolved antenna over the frequency range from $2.35 \mathrm{GHz}$ to $2.55 \mathrm{GHz}$. 


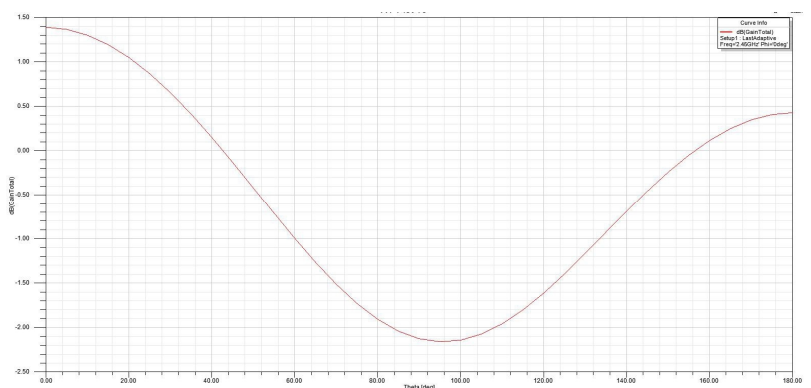

Fig. 5. Graph of the simulated gains over $-180^{\circ} \leq \theta \leq 180^{\circ}, \phi=0^{\circ}$ at $2.45 \mathrm{GHz}$ frequency.

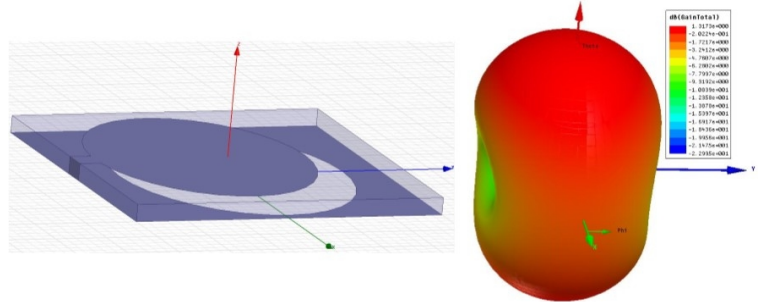

Fig. 6.Three dimensional radiation pattern at $2.45 \mathrm{GHz}$ frequency

The three dimensional radiation pattern over the range

$-180^{\circ} \leq \theta \leq 180^{\circ},-180^{\circ} \leq \phi \leq 180^{\circ}$ at $2.45 \mathrm{GHz}$ frequency is shown in Fig. 6 . We can see the antenna is omnidirectional one.

\section{Application of the evolved antennas}

One evolved antenna was mounted to a PC computer, see Fig. 7. It is verified that the evolved antenna works well.

\section{Conclusions}

This paper presents a small new patch antenna.

A new geometrical structure is proposed that the square substrate is given with side $30 \mathrm{~mm}$ (only about a quarter of the wavelength) and with thickness $1.6 \mathrm{~mm}$, and that an ellipse disc patch is loaded on the top of the substrate while the ground is cut off an ellipse disc on the bottom.

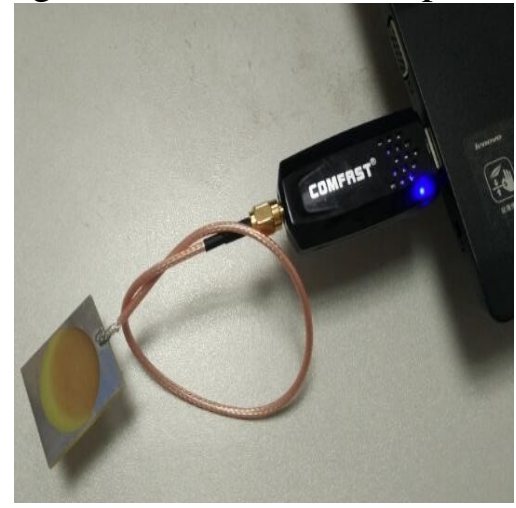

Fig. 7. The designed antenna used as a personal computer antenna in our lab.

A constrained optimization problem is formed for the antenna design with the sizes and the locations of the two ellipses and the width of the feed-line as solution vector. A DE is then used to solve the COP problem.

The evolved antenna has a relative band width approaching $10 \%$, while a usual similar antenna would be about $4 \%$ band width.

One antenna was mounted to a personal computer for indoor use. And we found they worked very well.

\section{Acknowledgements}

This work was supported by the National Natural Science Foundation of China and other foundations(No.s: 61271140, 61203306, 2012001202, 60871021). 


\section{References}

[1] Haupt Randy L., "Thinned arrays using genetic algorithms", IEEE Transactions on Antennas and Propagation, Vol. 42, No. 7, pp. 993999, July 1994.

[2] Linden D. S. and Altshuler E. E., "Automating wire antenna design using genetic algorithms", Microwave Journal, Vol. 39, No. 3, pp.74-86, 1996.

[3] Weile D.S., Michielssen E., "Genetic algorithm optimization applied to electromagnetics: a review", IEEE Trans. on Antennas and Propagation, Vol. 40, No. 3, pp. 343, 1997.

[4] Byun Gangil, Choo Hosung, Kim Sunwoo, "Design of a dual-band quadrifilar helix antenna using stepped-width arms", IEEE Transactions on Antennas and Propagation, Vol. 63, No. 4, p 1858-1862, April 1, 2015.

[5] Lohn Jason D., Linden Derek S., Blevins Bruce; Greenling Thomas, Allard Mark R., ”Automated synthesis of a lunar satellite antenna system", IEEE Transactions on Antennas and Propagation, Vol. 63, No. 4, pp. 1436-1444, April 1, 2015.

[6] Liu Bo, Aliakbarian Hadi, Ma Zhongkun, Vandenbosch Guy A.E.,Gielen Georges, Excell Peter, "An efficient method for antenna design optimization based on evolutionary computation and machine learning techniques", IEEE Transactions on Antennas and Propagation, Vol. 62, No. 1, pp. 7-18, January 2014.

[7] Deb Arindam, Roy Jibendu Sekhar, Gupta Bhaskar, "Performance comparison of differential evolution, particle swarm optimization and genetic algorithm in the design of circularly polarized microstrip antennas", IEEE Transactions on Antennas and Propagation, Vol. 62, No. 8, pp. 3920-3928, August 2014.

[8] Wen Ya-Qing, Wang Bing-Zhong, Ding Xiao," A Wide-Angle Scanning and Low Sidelobe Level Microstrip Phased Array Based on Genetic

Algorithm Optimization", IEEE Transactions on Antennas and Propagation, Vol. 64, No. 2, pp. 805-810, February 1, 2016.

[9] Gorman Philip J., Gregory Micah D., Werner Douglas H., "Design of ultra-wideband, aperiodic antenna arrays with the CMA evolutionary strategy", IIEEE Transactions on Antennas and Propagation, Vol. 62, No. 4, pp. 1663-1672, April 2014.

[10] El-Makadema Ahmed, Rashid Laith, Brown Anthony K., "Geometry design optimization of large-scale broadband antenna array systems", IIEEE Transactions on Antennas and Propagation, Vol. 62, No. 4, p 1673-1680, April 2014.

[11] Al-Rawi Ali, Hussain Ahmed, Yang Jian, Franzen Magnus, Orlenius Charlie, Kishk Ahmed A., "A new compact wideband MIMO antenna - The double-sided tapered self-grounded monopole array", IIEEE Transactions on Antennas and Propagation, Vol. 62, No. 6, p 33653369, June 2014.

[12] Haupt Randy L., "Adaptively thinned arrays", IIEEE Transactions on Antennas and Propagation, vol. 63, No. 4, pp. 1626-1632, April 1, 2015.

[13] Lema Gebrehiwet, Tesfamariam Gebremichael T., Mohammed Mohammed Ismail, "A novel elliptical-cylindrical antenna array for radar applications", IIEEE Transactions on Antennas and Propagation, Vol. 64, No. 5, pp. 1681-1688, May 2016.

[14] Kennedy J., Eberhart R.C., ’Swarm Intelligence”, Morgan Kaufmann, 2001.

[15] Boeringer Daniel W., Werner Douglas H., "Particle swarm optimization versus genetic algorithms for phased array synthesis", IIEEE Transactions on Antennas and Propagation, Vol. 52, No. 3, pp. 771-779, March 2004. 
[16] Xu Wanye, Duan B.Y., Li Peng, Hu Naigang, Qiu Yuanying, ”Multiobjective particle swarm optimization of boresight error and transmission loss for airborne radomes", IIEEE Transactions on Antennas and Propagation, Vol. 62, No. 11, pp. 5880-5885, November 1, 2014.

[17] Jang Chol-Hyon, Hu, Fei, He Feng, Li Jun, Zhu Dong, 'LowRedundancy Large Linear Arrays Synthesis for Aperture Synthesis Radiometers Using Particle Swarm Optimization", IIEEE Transactions on Antennas and Propagation, Vol. 64, No. 6, pp. 2179-2188, June 2016.

[18] Storn R., Price K., "Differential evolution - a simple and efficient heuristic for global optimization over continuous spaces”, Journal of Global Optimization, Vol. 11, pp. 341-359, 1997.

[19] Kurup Dhanesh G., Himdi Mohamed, Rydberg Anders, "Synthesis of Uniform Amplitude Unequally Spaced Antenna Arrays Using the Differential Evolution Algorithm", IIEEE Transactions on Antennas and Propagation, Vol. 51, No. 9, pp. 2210-2217, September 2003.

[20]Ding Yang, Jiao Yong-Chang, Zhang Li, Li Biao, "Solving port selection problem in multiple beam antenna satellite communication system by using differential evolution algorithm", IIEEE Transactions on Antennas and Propagation, Vol. 62, No. 10, pp. 5357-5361, October 1, 2014.

[21]Zhang Qingfu, Tsang Edward, Virginas Botond, "Expensive multiobjective optimization by MOEA/D with gaussian process model", IEEE Transactions on Evolutionary Computation, Vol. 14, No. 3, pp 456-474, June 2010. 